\title{
Endothelial function in a cardiovascular risk population with borderline ankle-brachial index
}

\author{
This article was published in the following Dove Press journal: \\ Vascular Health and Risk Management \\ 22 February 201I \\ Number of times this article has been viewed
}

\author{
Kari Syvänen' \\ Päivi Korhonen ${ }^{2}$ \\ Auli Partanen ${ }^{3}$ \\ Pertti Aarnio' \\ 'Department of Surgery, Satakunta \\ Hospital District, Pori, Finland; \\ ${ }^{2}$ Central Satakunta Health Federation \\ of Municipalities, Harjavalta, Finland; \\ ${ }^{3}$ Department of Biostatistics, \\ University of Turku, Turku, Finland
}

Introduction: The diagnosis of peripheral arterial disease (PAD) can be made by measuring the ankle-brachial index (ABI). Traditionally ABI values $>1.00-1.40$ have been considered normal and $\mathrm{ABI} \leq 0.90$ defines PAD. Recent studies, however, have shown that individuals with ABI values between $0.90-1.00$ are also at risk of cardiovascular events. We studied this cardiovascular risk population subgroup in order to determine their endothelial function using peripheral arterial tonometry (PAT).

Methods: We selected 66 individuals with cardiovascular risk and borderline ABI. They all had hypertension, newly diagnosed glucose disorder, metabolic syndrome, obesity, or a ten year risk of cardiovascular disease death of 5\% or more according to the Systematic Coronary Risk Evaluation System (SCORE). Subjects with previously diagnosed diabetes or cardiovascular disease were excluded. Endothelial function was assessed by measuring the reactive hyperemia index (RHI) from fingertips using an Endo-PAT device.

Results: The mean ABI was 0.95 and mean RHI 2.11. Endothelial dysfunction, defined as RHI $<1.67$, was detected in 15/66 (23\%) of the subjects. There were no statistically significant differences in RHI values between subjects with different cardiovascular risk factors. The only exception was that subjects with impaired fasting glucose (IFG) had slightly lower RHI values (mean RHI 1.91) than subjects without IFG (mean RHI 2.24) $(P=0.02)$.

Conclusions: In a cardiovascular risk population with borderline ABI nearly every fourth subject had endothelial dysfunction, indicating an elevated risk of cardiovascular events. This might point out a subgroup of individuals in need of more aggressive treatment for their risk factors.

Keywords: peripheral arterial disease, ankle-brachial index, cardiovascular risk, endothelial dysfunction

\section{Introduction}

Peripheral arterial disease (PAD) is a manifestation of atherosclerosis in the lower limbs. It can be diagnosed by measuring the ankle-brachial index (ABI). A value of $\leq 0.90$ determines PAD with a sensitivity of $95 \%$, and a specificity of almost $100 \%{ }^{1}$ Traditionally normal ABI is considered as having a value of $>1.00 .^{2} \mathrm{ABI}$ values between 0.90 and 1.00 are also associated with an inversely related risk of cardiovascular outcomes. ${ }^{3}$ Therefore individuals with so called "borderline ABI" have an elevated risk of cardiovascular events. ${ }^{4}$ Their risk is lower than in patients with PAD, but still significant, and they have increased risk of developing PAD without interventions. ${ }^{5}$

Although endothelium is constructed from a monolayer of rather simple cells, it performs many important homeostatic functions. The role of endothelial function in 
cardiovascular disease is well established and individuals with endothelial dysfunction have an increased risk of cardiovascular events. ${ }^{6,7}$ Since the identification of nitric oxide, many different methods have been used to detect endothelial function, but due to logistical limitations their routine use in risk detection has been low. ${ }^{8}$ The gold standard technique is flow-mediated dilation (FMD) of the brachial artery. ${ }^{9,10}$ Novel digital pulse amplitude tonometry (PAT) offers the possibility of an easy and rapid assessment of vascular function. ${ }^{11-13}$ Recently it has been demonstrated that abnormalities in pulse wave amplitude are correlated with flow mediated dilatation measurement results. ${ }^{12}$

We used this novel method to determine the prevalence of endothelial dysfunction in a cardiovascular risk population with borderline ABI.

\section{Materials and methods}

The present study is a sub-study of the Harmonica project, and a detailed description of the enrolment and examination methods has been published earlier. ${ }^{14}$ In the Harmonica project we measured the ABI of 972 individuals with newly diagnosed hypertension, glucose disorders, metabolic syndrome, obesity, or a ten year risk of cardiovascular disease death of $5 \%$ or more according to the Systematic Coronary Risk Evaluation System (SCORE). None of them had been previously diagnosed with cardiovascular disease or diabetes before participating in the Harmonica project. In this study we selected 66 individuals with borderline ABI and measured their endothelial function using PAT. This was a small sample population to test this novel methodology in this setting in the preliminary study.

ABI was measured using a blood pressure cuff and a Doppler instrument (UltraTec ${ }^{\circledR}$ PD1v with a $5 \mathrm{MHz}$ vascular probe, United Kingdom) with the subject in a supine position. We measured the systolic blood pressure (SBP) of the left and right brachial arteries in the upper arms. In the lower limbs, both the dorsalis pedis arterial pressure and posterior tibial arterial pressure were measured if available. The cuff was placed just above the level of the malleoli. A trained nurse performed all measurements. The ABI was the higher SPB of the lower limbs divided by the higher of the brachial SBPs. Participants who had an ABI value of $\leq 0.90$ in either leg were categorized as having PAD. Subjects with an ABI value of between 0.91 and 1.00 were considered as borderline PAD. ABI > 1.00-1.40 was considered normal.

Endothelial function was assessed using a Endo-PAT device (Itamar Medical Ltd, Caesarea, Israel). During the measurement the subject sat in a chair with their hands at the level of their heart and fingers hanging freely. Fingertip probes were placed on both index fingers and pulse wave amplitudes were detected and recorded during the study. After a five-minute baseline measurement, arterial flow was occluded using a cuff on the non-dominant arm. The cuff was inflated to $40 \mathrm{mmHg}$ above systolic pressure. After five minutes of occlusion, the cuff was rapidly deflated to allow reactive hyperemia. Pulse wave amplitudes were recorded again for at least five minutes. The software provided by the manufacturer was then used to compare the arterial pressure ratio in the two fingers before and after occlusion. It then calculated a reactive hyperemia index (RHI), which was a ratio of the average pulse wave amplitude measured over 60 seconds, starting one minute after cuff deflation, to the average pulse wave amplitude measured at the baseline. The other arm served as a control and the ratio was corrected for changes in the systemic vascular tone. An RHI value of $<1.67$ was used as a cut-off value to diagnose endothelial dysfunction. ${ }^{13}$

\section{Statistical analysis}

The statistical significance of differences between frequency distributions was tested using the Pearson's $\chi^{2}$ test. The difference between mean values was tested using the pairwise $t$-test or the Wilcoxon rank sum test for two samples. The normality of residuals was tested with the Shapiro-Wilks test, and if residuals were not normally distributed the difference between RHI values was tested using the Wilcoxon test. $P$-values of less than 0.05 were considered statistically significant. The statistical analyses were carried out using SAS/STAT (r) software, Version 9.2 of the SAS System for Windows (SAS Institute Inc, Cary, NC, USA).

All of the participants provided written informed consent for the project and subsequent medical research. The study protocol and consent forms were reviewed and approved by the ethics committee of Satakunta Hospital District.

\section{Results}

The mean age in this study group was 60.1 years $(50-75)$. We studied 66 subjects, 26 (39\%) were males and $40(61 \%)$ females. Ten (15\%) were current smokers and $21(32 \%)$ former smokers. Hypertension was diagnosed in 35 individuals (55\%), 27 (41\%) had impaired fasting glucose (IFG), 22 (33\%) impaired glucose tolerance (IGT), and six were diabetics $(9 \%)$ (Table 1). More than one cardiovascular risk factor was found in 36 subjects (55\%).

The mean (SD) ABI was $0.95(0.023)$ and the mean (SD) RHI 2.11 (0.552). Endothelial dysfunction, defined 
Table I Characteristics of the study subjects

\begin{tabular}{lll}
\hline Mean age (range) years & 60.1 & $(50-75)$ \\
Males (\%) & 26 & $(39)$ \\
Females (\%) & 40 & $(6 \mathrm{I})$ \\
Current smoker (\%) & 10 & $(15)$ \\
Former smoker (\%) & 21 & $(32)$ \\
Hypertension (\%) & 35 & $(55)$ \\
IFG (\%) & 27 & $(41)$ \\
IGT (\%) & 22 & $(33)$ \\
T2DM (\%) & 6 & $(9)$ \\
Mean ABI (SD) & 0.95 & $(0.023)$ \\
Mean RHI (SD) & 2.11 & $(0.552)$ \\
Endothelial dysfunction (\%) & 15 & $(23)$ \\
\hline
\end{tabular}

Abbreviations: $A B I$, ankle-brachial index; IFG, impaired fasting glucose; IGT, impaired glucose tolerance; $\mathrm{RHI}$, reactive hyperemia index.

as RHI $<1.67$, was detected in 15/66 (23\%). When we compared the RHI values of subjects with different risk factors, no statistically significant differences were found. The only exception was that subjects with IFG had slightly lower RHI values (mean RHI 1.91) than subjects without IFG (mean RHI 2.24) (Table 2).

\section{Discussion}

The endothelium plays an important role in the regulation of vascular tone and structure as first recognized by the experiments of Robert Furchgott's group. ${ }^{15}$ The endothelium is also a target of vascular inflammation and thrombosis, the key events of the atherosclerotic disease process and its clinical implications, such as myocardial infarction and stroke. With the presence of cardiovascular risk factors such as hypercholesterolemia, hypertension, and smoking, the endothelium has reduced nitric oxide availability, impairing its function and endothelium-dependent vasodilation. ${ }^{16}$ This endothelial dysfunction is associated with a risk of major cardiovascular events. ${ }^{17}$ In the study carried out by Hamburg et al (2008), male sex, body mass index, ratio of total to high density lipoprotein cholesterol, diabetes mellitus, smoking, and lipid-lowering treatment were inversely related to the PAT ratio. ${ }^{18}$ An improvement in endothelial function and a reduction in the risk of vascular events can be achieved by intensively treating risk factors such as hypertension. ${ }^{19-21}$

For decades, ABI has been used as a tool of vascular practice to quantify the severity of occlusive disease among patients with leg symptoms, and to decide the need for vascular interventions. More recently the role of ABI has been expanded, as it is a marker of cardiovascular risk. A normal ABI value is between 1.00-1.40 and PAD is defined as an ABI value of 0.90 or less. ABI $>1.40$ might indicate medial sclerosis. The presence of an abnormal ABI value increases the risk of myocardial infarction and death, among other adverse outcomes. ${ }^{5,22,23}$ In the Walking and Leg Circulation Study (WALCS), subjects with low normal (ABI 1.00-1.09) and borderline PAD (ABI 0.90-0.99) also had a significant risk of mobility loss during follow up. ${ }^{24}$ The likely mechanism was progression towards PAD. We have previously shown that almost a third of hypertensive patients without known diabetes or prior cardiovascular or renal diseases have asymptomatic PAD or borderline PAD. ${ }^{25}$ These results force us to rethink the optimal interpretation of $\mathrm{ABI}$ in vascular practices, and to start interventions early enough.

We studied a cardiovascular risk population that had not been previously diagnosed with diabetes or cardiovascular or renal diseases. We took a small sample of subjects with borderline ABI to evaluate the prevalence of endothelial dysfunction in this group and to test the methodology. To our knowledge this is the first study in this kind of setting. The homogenous nature of this group may be the main reason why we did not find differences in RHI values between different types of risk-factors (ie, all subjects had at least one cardiovascular risk factor). We used the cut-off value RHI $<1.67$, determined in previous studies, to diagnose endothelial dysfunction. ${ }^{26-28}$ Almost every fourth subject with borderline ABI had endothelial dysfunction. This may point to a subgroup of patients who need intensive risk factor management in order to prevent cardiovascular events and loss of mobility.

Table 2 Comparison between subjects with different risk factors and RHI values

\begin{tabular}{|c|c|c|c|c|}
\hline Risk factor & $\begin{array}{l}\text { Number of subjects } \\
\text { with risk factor }\end{array}$ & $\begin{array}{l}\text { Mean RHI (SD) } \\
\text { (risk factor) }\end{array}$ & $\begin{array}{l}\text { Mean RHI (SD) } \\
\text { (no risk factor) }\end{array}$ & P-value \\
\hline IGT and/or IFG and/or T2DM & 55 & I.91 (0.332) & $2.25(0.587)$ & 0.16 \\
\hline T2DM & 6 & $1.79(0.156)$ & $2.14(0.568)$ & 0.14 \\
\hline IGT & 22 & $2.13(0.390)$ & $2.08(0.632)$ & 0.30 \\
\hline IFG & 27 & I.9I (0.397) & $2.24(0.608)$ & $0.02^{\mathrm{a}}$ \\
\hline Hypertension & 35 & $2.04(0.44 I)$ & $2.18(0.656)$ & 0.70 \\
\hline Smoking & 10 & $2.04(0.643)$ & $2.12(0.531)$ & 0.62 \\
\hline
\end{tabular}

aStatistically significant.

Abbreviations: IFG, impaired fasting glucose; IGT, impaired glucose tolerance; RHI, reactive hyperemia index; T2DM, type 2 diabetes mellitus; SD, standard deviation. 
PAT testing is free of operator dependent errors, because results are analyzed with a computerized, automated algorithm. The observations can be considered as being free of subjective bias when compared to flow mediated dilation measurements with ultrasound from brachial arteries. This reliability is the strength of the method we used. In contrast, the reproducibility of the ABI measurements can be questioned if they are performed by inexperienced personnel. In the study carried out by Mätzke et al (2003), $16 \%$ of $\mathrm{ABI}$ values differed by 0.15 or more from the median when measurements were taken by doctors with little or no instructions. ${ }^{29}$ They concluded that to obtain reproducibility and quantitative measurement values, the measurements have to be performed by trained personnel. Our measurements were taken by a trained nurse. There is also a so-called center effect when ABI is measured with a sphygmomanometer and a Doppler probe but not with a semiautomatic device. ${ }^{30}$ This should be taken into account when planning multi-center studies based on ABI measurement.

Although the PAT results are free of intra- and interobserver variation, this method may be influenced by changes in the physiological state of the study subject. We made all the measurements in a controlled environment, including a quiet, darkened room at a steady room temperature. The other arm served as an internal control. Because dietary intake may influence the results, all the measurements were taken with the subject in a fasting state. ${ }^{31,32}$ Obviously a small number of study subjects can be considered as a limitation to our study, but the study group is a representative sample of people at cardiovascular risk in the general population.

Now that there is growing evidence to link low RHI scores and the risk of cardiovascular disease, it is encouraging that we can use this non-invasive method to provide an additional level of risk stratification to initiate early and aggressive treatments to prevent future cardiovascular events. As pointed out by Celermajer (2008), the exact role of PAT in this regard is not clear, but there is evidence that it gives relevant predictive and prognostic data about endothelial function. ${ }^{33}$ However, the prognostic usefulness of this technique in clinical practice is only beginning to emerge. The technique per se is suitable for clinical use because it is reasonably priced, non-invasive and it requires minimal training. In the future it will be necessary to perform large-scale clinical studies to provide us with further information about the diagnostic usefulness of PAT in everyday clinical practice. There might still be unknown factors to be discovered that influence PAT measurements and their results. Some clinical studies are currently under way and we can expect results in the near future.

\section{Disclosure}

No conflicts of interest were declared in relation to this paper.

\section{References}

1. Dormandy JA, Rutherford RB. Management of peripheral artery disease (PAD). TASC Working Group. Trans Atlantic Inter-Society Consensus (TASC). J Vasc Surg. 2000;31:S1-S44.

2. Fowkes FGR. The measurement of atherosclerotic peripheral arterial disease in epidemiological surveys. Int J Epidemiol. 1988;17:248-254.

3. Lamina C, Meisinger C, Heid IM, et al. Association of ankle-brachial index and plaques in the carotid and femoral arteries with cardiovascular events and total mortality in a population-based study with 13 years of follow-up. Eur Heart J. 2006;27:2580-2587.

4. Diehm C, Lange S, Darius H, et al. For the getABI Study Group. Association of low ankle brachial index with high mortality in primary care. Eur Heart J. 2006;27:1743-1749.

5. Fowkes FGR, Murray GD, Newman AB, et al. Ankle brachial index combined with Framingham risk score to predict cardiovascular events and mortality. A meta-analysis. JAMA. 2008;300:197-208.

6. Windlansky ME, Gokce N, Keaney JF, et al. The clinical implications of endothelial dysfunction. J Am Coll Cardiol. 2003;42:1149-1160.

7. Yeboah J, Crouse JR, Hsu FC, et al. Brachial flow-mediated dilation predicts incident cardiovascular events in older adults: the Cardiovascular Health Study. Circulation. 2007;115:2390-2397.

8. McMackin CJ, Vita JA. Update on nitric oxide-dependent vasodilation in human subjects. Methods Enzymol. 2005;396:541-553.

9. Agewall S, Doughty RN, Bagg W, et al. Comparison of ultrasound assessment of flow-mediated dilation in the radial and brachial artery with upper and forearm cuff positions. Clin Physiol. 2001;21:9-14.

10. Corretti MC, Anderson TJ, Benjamin EJ, et al. International brachial artery reactivity task force. Guidelines for the ultrasound assessment of endothelial dependent flow mediated vasodilatation of the brachial artery. J Am Coll Cardiol. 2002;39:257-265.

11. Faizi AK, Kornmo DW, Agewall S. Evaluation of endothelial function using finger plethysmography. Clin Physiol Funct Imaging. 2009;29:372-375.

12. Kuvin JT, Patel AR, Sliney KA, et al. Assessment of peripheral vascular endothelial function with finger arterial pulse wave amplitude. Am Heart J. 2003;146:161-172.

13. Bonetti PO, Pumper GM, Higano ST, et al. Non-invasive identification of patients with early coronary atherosclerosis by assessment of digital reactive hyperemia. J Am Coll Cardiol. 2004;44:2137-2141.

14. Korhonen P, Aarnio P, Saaresranta T, et al. Glucose homeostasis in hypertensive subjects. Hypertension. 2008;51:945-949.

15. Furchgott RF, Zawadzki JV. The obligatory role of endothelial cells in the relaxation of arterial smooth muscle by acetylcholine. Nature. 1980;288:373-376.

16. Giannotti G, Landmesser U. Endothelial dysfunction as an early sign of atherosclerosis. Herz. 2007;32:568-572.

17. Lerman A, Zeiher AM. Endothelial function. Cardiac events. Circulation. 2005;111:363-368.

18. Hamburg NM, Keyes MJ, Larson MG, et al. Cross-sectional relations of digital vascular function to cardiovascular risk factors in the Framingham heart study. Circulation. 2008;117:2467-2474.

19. Modena MG, Bonetti L, Coppi F, et al. Prognostic role of reversible endothelial dysfunction in hypertensive postmenopausal women. $\mathrm{J} \mathrm{Am}$ Coll Cardiol. 2002;40:505-510.

20. Iwatsubo H, Nagano M, Sakai T, et al. Converting enzyme inhibitor improves forearm reactive hyperemia in essential hypertension. Hypertension. 1997;29:286-290.

21. Giugliano D, Marfella R, Acampora R, et al. Effects of perindopril and carvedilol on endothelium-dependent vascular functions in patients with diabetes and hypertension. Diabetes Care. 1998;21:631-636. 
22. Lee AJ, Price JF, Russell MJ, et al. Improved prediction of fatal myocardial infarction using the ankle brachial index in addition to conventional risk factors: the Edinburgh Artery Study. Circulation. 2004;110:3075-3080.

23. Criqui MH, Langer RD, Fronek A, et al. Mortality over a period of 10 years in patients with peripheral arterial disease. $N$ Engl J Med. 1992;326:381-386.

24. McDermott MM, Guralnik JM, Tian L, et al. Associations of borderline and low normal ankle-brachial index values with functional decline at 5-year follow up. J Am Coll Cardiol. 2009:53;1056-1062.

25 Korhonen PE, Syvänen KT, Vesalainen RK, et al. Ankle-brachial index is lower in hypertensive than in normotensive individuals in a cardiovascular risk population. J Hypertension. 2009;27:2036-2043.

26. Yeo TW, Lampah DA, Gitawati R, et al. Impaired nitric oxide bioavailability and L-arginine reversible endothelial dysfunction in adults with falciparum malaria. J Exp Med. 2007;204:2693-2704.

27. Yinon D, Lowenstein L, Suraya S, et al. Pre-eclampsia is associated with sleep-disordered breathing and endothelial dysfunction. Eur Respir J. 2006;27:328-333.
28. Ohno Y, Hashiguchi T, Maenosono R, et al. The diagnostic value of endothelial function as a potential sensor of fatigue in health. Vasc Health Risk Manag. 2010;24:135-144.

29. Mätzke S, Franckena M, Aalbäck A, et al. Ankle brachial index measurements in critical leg ischaemia - the influence of experience on reproducibility. Scand J Surg. 2003;92:144-147.

30. Vierron E, Halimi J, Tichet J, et al. Centre effect on ankle-brachial index measurement when using the reference method (Doppler and manometer): results from a large cohort study. Am J Hypertension. 2009;22:718-722.

31. Giannattasio C, Zoppo A, Gentile G, et al. Acute effect of high-fat meal on endothelial function in moderately dyslipidemic subjects. Arterioscler Thromb Vasc Biol. 2005;25:406-410.

32. Gaenzer H, Sturm W, Neumayr G, et al. Pronounced postprandial lipemia impairs endothelium-dependent dilation of the brachial artery in men. Cardiovasc Res. 2001;52:509-516.

33. Celermajer DS. Reliable endothelial function testing: at our fingertips? Circulation. 2008;117:2428-2430.
Vascular Health and Risk Management

\section{Publish your work in this journal}

Vascular Health and Risk Management is an international, peer-reviewed journal of therapeutics and risk management, focusing on concise rapid reporting of clinical studies on the processes involved in the maintenance of vascular health; the monitoring, prevention and treatment of vascular disease and its sequelae; and the involvement of metabolic

\section{Dovepress}

disorders, particularly diabetes. This journal is indexed on PubMed Central and MedLine. The manuscript management system is completely online and includes a very quick and fair peer-review system, which is all easy to use. Visit http://www.dovepress.com/testimonials.php to read real quotes from published authors.

Submit your manuscript here: http://www.dovepress.com/vascular-health-and-risk-management-journal 